\title{
LINFONODO SENTINELA: IMPORTÂNCIA NA CIRURGIA DO CÂNCER GÁSTRICO E PERSPECTIVA DA APLICAÇÃO DE UM MODELO EXPERIMENTAL EM CANINOS
}

\author{
Sentinel lymph node: importance in gastric cancer surgery and perspective of the application of \\ an experimental model in canine
}

José Ricardo de Moura Torres de MELO, Luiz Gonzaga Porto PINHEIRO

Trabalho realizado no Laboratório de Cirurgia Experimental Prof. Dr. Saul Goldenberg/GEEON (Grupo de Educação e Estudo Oncológico) - extensão do Departamento de Cirurgia da Faculdade de Medicina da Universidade Federal do Ceará, Fortaleza, CE, Brasil

DESCRITORES - Câncer gástrico. Metástase linfática. Biópsia de linfonodo sentinela.

\section{Correspondência:}

José Ricardo de Moura Torres Rua Pe. Valdevino, 1870 - Aldeota 60135-041 - Fortaleza, CE - Brasil

Fonte de financiamento: não há Conflito de interesses: não há

Recebido para publicação: 06/07/2010 Aceito para publicação: 23/06/2010

HEADINGS - Gastric cancer. Node metastasis. Sentinel lymph node biopsy.
RESUMO - Introdução - A única perspectiva de cura do câncer gástrico ainda é o tratamento cirúrgico - gastrectomia associada com linfadenectomia ampliada -, embora tal procedimento possa levar a alto índice de morbidade ou mortalidade. Atualmente, a pesquisa do linfonodo sentinela para o câncer gástrico inicial (EC T1/ T2) apresenta argumentos que podem está modificando esta conduta. Objetivo - Realizar revisão bibliográfica atualizada da importância do linfonodo sentinela na cirurgia do câncer gástrico e apresentar modelo experimental, em caninos, que poderia se prestar como treinamento de cirurgiões para o método da pesquisa de linfonodo sentinela em estômago. Método - Revisão da literatura baseaa no Pubmed/Medline, Scielo e Lilacs cruzando os unitermos câncer gástrico, metástase linfática e biópsia de linfonodo sentinela e apresentação de método experimental para localização de nódulo sentinela. Conclusões - As evidências demonstram que há muito para se comprovar, entretanto a definição do tratamento do $\mathrm{CaG}$ nos tumores T1 parece ser o maior foco e abre bastante espaço para pesquisa. $\mathrm{O}$ modelo experimental em caninos para a pesquisa do LS no estômago, poderia se prestar como treinamento para cirurgiões na aplicação do método.

ABSTRACT - Introduction - The only possibility to cure gastric cancer is still through a surgical treatment - gastrectomy associated with extended lymphadenectomy -, although this procedure may lead to high rates of morbidity or mortality. Currently, sentinel lymph node biopsy for early gastric cancer (T1/T2 EC) presents arguments that can change this behavior. Aim - To review updated bibliography of the importance of sentinel lymph nodes in gastric cancer surgery and present experimental model in dogs, which could provide training for surgeons as to the method of sentinel lymph node in the stomach. Method - Review of literature basing on Pubmed / Medline, Lilacs and Scielo crossing the key-words gastric cancer, lymph node metastasis and sentinel lymph node biopsy and presenting an experimental method for localization of sentinel node. Conclusions - The evidence shows that there is much to prove, however, the definition of the treatment of gastric cancer in T1 tumors appears to be the major focus and opens up plenty of space for research. The experimental model in dogs for the study of LS in the stomach, could provide training for surgeons in the method.

\section{INTRODUÇÃO}

$\mathrm{O}$ câncer gástrico $(\mathrm{CaG})$ é atualmente a quarta neoplasia maligna mais frequente e a segunda causa de morte entre todos os tipos de câncer no mundo. Sua maior incidência é no Japão, onde se encontram 780 casos por 100.000 habitantes. No Brasil, por 100.000 habitantes, existem de 10 a 20 casos na população masculina e de cinco a 10 casos na população feminina ${ }^{21}$.

A gastrectomia (total ou parcial) associada com linfadenectomia é a principal alternativa terapêutica e representa, atualmente, a única perspectiva de cura da doença. Disseminações por contiguidade e hematogênica são de difícil controle. A linfática pode ser controlada com eficiência através de linfadenectomia ampliada, pois o status linfonodal é um indicador prognóstico 
importante ${ }^{1,9,14}$. A linfadenectomia ampliada sofre restrições, pois em muitos casos que foi executada mostra linfonodos livres de neoplasia tendo este procedimento significativo risco de morbimortalidade ${ }^{4,25}$.

O estudo pré-operatório por imagens (PET CT, US convencional e endoscópico ou ressonância magnética) tem ainda limitações na detecção de metástase linfonodal para eventual planejamento cirúrgico ${ }^{27,35}$.

A partir do ano 2000 surgiram publicações sobre o mapeamento linfático (linfocintilografia) e pesquisa do linfonodo sentinela (LS) no tratamento do CaG, que aplicados a estes tumores representam, atualmente, tentativa de aperfeiçoar a capacidade de detecção das metástases linfonodais e orientar terapêutica individualizada ${ }^{18,23,4,25,38}$.

O principio da pesquisa do LS no CaG baseia-se na premissa de que, na ausência de metástase linfonodal (N0), procedimentos menos radicais (laparoscópicos ou endoscópicos) poderiam ser realizados diminuindo a morbidade e mortalidade cirúrgicas $8,10,13,22,27,32,41$.

É necessário atentar-se para os resultados falsonegativos que, em algumas situações, ocorrem devido à obstrução de vasos linfáticos nos tumores mais avançados (EC T3/T4) apresentando disseminação linfática atípica. Outra situação que merece cuidado é a existência da metástase salteada (jumping metastasis) que pode ocorrer em 15 a $20 \%$ dos casos. São linfonodos situados em cadeias distantes do tumor primário (N2-N3) que ultrapassariam a cadeia perigástrica (N1). Alguns autores sugerem que nestes casos, além da drenagem linfática atípica, a neoplasia poderia ter sido subestadiada, ou seja, com tamanho e profundidade maiores e invasão, pelo menos, de camada submucosa ${ }^{12}$.

Parece ser consenso que a pesquisa do LS no $\mathrm{CaG}$ deva ser feita em tumores na fase inicial com EC $\mathrm{T}_{1} \mathrm{NO}^{27}$, conduta esta já adotada em neoplasias da mama e melanomas.

\section{MÉTODO}

Revisão da literatura baseada no Pubmed/ Medline, Scielo e Lilacs cruzando os unitermos câncer gástrico, metástase linfática e biópsia de linfonodo sentinela e apresentação de método experimental para localização de nódulo sentinela.

\section{Linfonodo sentinela - histórico e manuseio}

A drenagem linfática no planejamento terapêutico do câncer data de meados do século XVIII quando Henry François Le Dran o fez para o câncer de mama (CaM) descrevendo sua progressão linfática. Baseado nestes estudos Halsted, no século XIX, desenvolveu a primeira perspectiva de cura para o câncer de mama, ou seja, mastectomia com ressecção dos músculos peitorais associado à linfadenectomia axilar com ressecção em monobloco. Esta estratégia cirúrgica que foi ampliada a outros tumores malignos (melanoma, neoplasia uterinas, gástricas, intestinais) perdurou por um século ${ }^{36,37}$.Até 1969 Veronesi seguia rigorosamente esta conduta para o tratamento desse tumor ${ }^{39}$. Entretanto nos anos seguintes este grupo demonstrou a viabilidade da ressecção mamária parcial com linfadenectomia axilar associada à rádio e quimioterapia adjuvantes, modificando radicalmente a cirurgia no tratamento do câncer de mama. Observou também os esvaziamentos axilares desnecessários em paciente com axila negativa e mostrou ainda a progressão escalonada da colonização dos linfonodos axilares nas neoplasias mamárias (nível um, dois e três) sustentando controvérsias do tratamento para este tumor ${ }^{5,6}$.

O termo linfonodo sentinela (LS) foi proposto inicialmente em 1977 por Cabanas que, usando linfangiograma, estudou a disseminação linfática do carcinoma de pênis e atribuiu a denominação LS ao primeiro linfonodo que fazia a drenagem deste tumor ao nível da arcada inguinal. A identificação e retirada deste linfonodo minimizaria a mutilação de um esvaziamento inguinal bilateral caso o mesmo fosse negativo. Porém a identificação de vias alternativas para disseminação linfática invalidou o pensamento da linfadenectomia seletiva ${ }^{7}$.

LS é o primeiro linfonodo a receber a drenagem linfática de um tumor e que pode ser detectado após injeção peritumoral de marcadores (Figura 1).

Linfonodos regionais

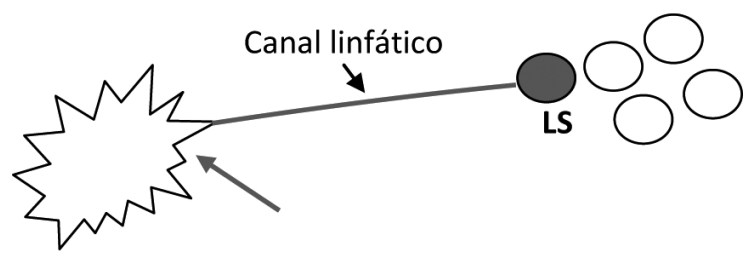

Tumor Injeção de marcadores

FIGURA 1 - Visão esquemática da técnica de mapeamento e posição de um linfonodo sentinela

Morton et al. ${ }^{29}$ desenvolveram modelo experimental injetando o corante azul isosulfan em pata de felinos para a pesquisa do LS na drenagem linfática da pele destes animais estimulando novamente a viabilidade da linfadenectomia seletiva. No ano seguinte este grupo demonstrou a importância e os detalhes técnicos do mapeamento linfático usando corantes na identificação do LS em melanoma de extremidades $15,29,30$.

Nos anos seguintes alguns autores publicaram trabalhos demonstrando resultados efetivos com 0 uso de substância radioativa (Tecnécio) e sua captação com o aparelho Gama Probe na identificação do LS em câncer de mama e melanomas na fase inicial ${ }^{2,3,26}$.

Atualmente o uso combinado de marcadores corantes e radioativos estão clinicamente estabelecidos na cirurgia da mama e melanoma em fase inicial (EC 
T1/T2) para verificação de metástases linfonodais e estadiamento definitivo destes tumores ${ }^{17,34}$.

É atribuído a Mikulics (1889) a idéia inicial da linfadenectomia extensa no CaG que na época declarou ser necessária também a remoção do pâncreas distal. Esta linfadenectomia extensa (D2) é ainda hoje defendida com vigor por vários autores e caracteriza-se como padrãoouro no tratamento cirúrgico no $\mathrm{CaG}$ com perspectiva de cura $^{9,14}$. Porém, tal definição pode estar mudando especialmente nos tumores na fase inicial EC T1/T211,27,33.

A pesquisa do LS para tumores malignos vem desenvolvendo-se em larga extensão e nos mais variados tipos de câncer tais como mama, vulva, colo uterino, cabeça e pescoço, trato urinário e aparelho digestório $16,17,28,40$. O comprometimento linfonodal é um dos mais importantes preditivos na sobrevida dos pacientes portadores de CaG. Neoplasias avançadas (EC T4) que cursam com linfonodos negativos parametastáticos, evidenciam melhor prognóstico ${ }^{1,14}$.

Independente de orientar ou não a extensão do procedimento cirúrgico a pesquisa do LS aumentaria a precisão do diagnóstico histológico deste linfonodo pelo o maior número de cortes seriados e da possibilidade de estudo imunoistoquímico, o que seria inviável para toda cadeia linfática. Linfonodos resultantes de linfadenectomia extensa, por serem estudados mais superficialmente, poderiam deixar escapar micrometástases ou colonizações mínimas detectáveis por métodos imunoistoquímicos levando ao subestadiamento linfonodal. Isto pode ocorrer mesmo naqueles pacientes que foram considerados livres de linfonodos metastáticos por exames histopatológicos de rotina, sugerindo que no $\mathrm{CaG}$ a extensão da doença é mais avançada que a indicada pelo resultado dos exames comumente realizados ${ }^{25,31}$.

A literatura mostra inúmeros trabalhos principalmente provenientes do Japão que já validam a gastrectomia modificada com linfadenectomia seletiva para o CaG em fase inicial EC T1/T2 $19,20,33$

Ichikura et al. ${ }^{19,20}$ utilizando Tecnécio (99mTc) e corante indocianina verde em pacientes portadores de CaG com EC T1NOMO demonstrou que é possível a realização de procedimento cirúrgico gástrico segmentar com linfadenectomia regionais em pacientes com biópsia de LS negativa, realizada por congelação, durante o procedimento (Figura 2).
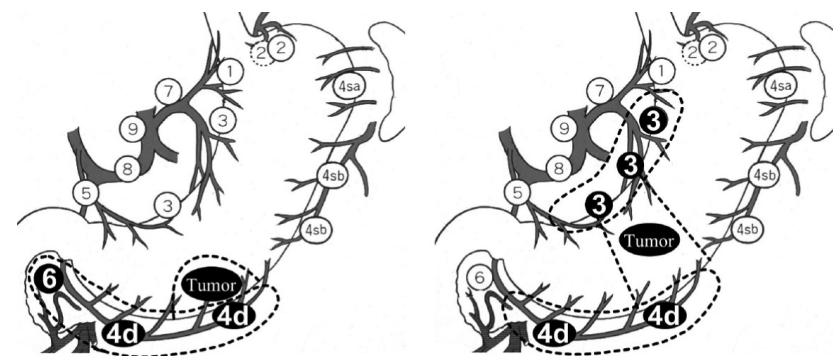

FIGURA 2 - Representação gráfica de operação realizada por Takashi Ichikura ${ }^{41}$
Em nosso meio, modelos experimentais estão sendo realizados com a finalidade de aprofundar e compreender a pesquisa de LS no estômago. Torres de $\mathrm{Melo}^{37}$, elaborou e viabilizou pesquisa em laboratório de LS no estômago (antro) de cães utilizando Tecnécio e corante azul patente com achados que lembram muito os LS encontrados no CaG (antro), ou seja, estação linfonodal 3 e 6 (Figura 3).

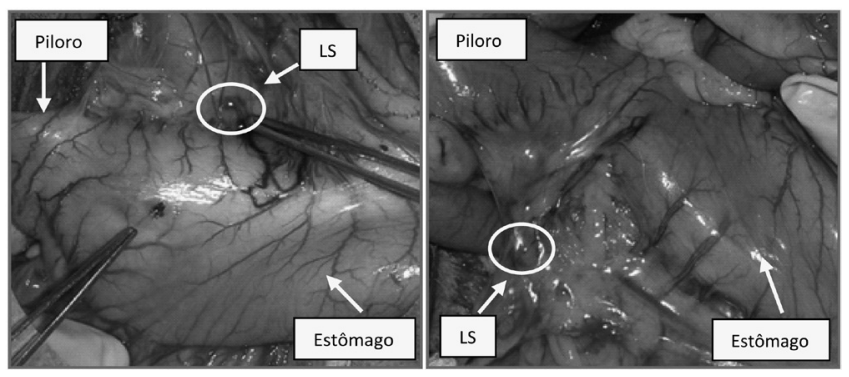

FIGURA 3 - À esquerda LS da pequena curvatura; à direita LS infrapilórico

As evidências demonstram que há muito para se comprovar, entretanto a definição do tratamento do CaG nos tumores T1 parece ser o maior foco e abre bastante espaço para pesquisa.

O modelo experimental em caninos para a pesquisa do LS no estômago, descrito acima, poderia se prestar como treinamento para cirurgiões na aplicação do método.

\section{REFERÊNCIAS}

1. Adachi Y, Oshiro T, Mori M, Maehara Y, Sugimachi K. Prediction of early and late recurrence after curative resection for gastric carcinoma. Cancer. 1996 Jun 15;77(12):2445-8.

2. Alex JC, Krag DN. Gamma-probe guided localization of lymph nodes. Surg Oncol. 1993;2(3):137-43.

3. Alex JC, Weaver DL, Fairbank JT, Rankin BS, Krag DN. Gammaprobe-guided lymph node localization in malignant melanoma. Surg Oncol. 1993 Oct;2(5):303-8.

4. Bonenkamp JJ, Hermans J, Sasako M, van de Velde CJ, Welvaart $\mathrm{K}$, Songun I, et al. Extended lymph-node dissection for gastric cancer. N Engl J Med. 1999 Mar 25;340(12):908-14.

5. Bucalossi P, Veronesi U, Pizzocaro G, Zucali R. [Results of preoperative radiotherapy of T-3 mammary carcinoma]. Tumori. 1972 Jul-Aug;58(4):203-11.

6. Bucalossi P, Veronesi U, Zingo L, Cantu C. Enlarged mastectomy for breast cancer. Review of 1,213 cases. Am J Roentgenol Radium Ther Nucl Med. 1971 Jan;111(1):119-22.

7. Cabanas RM. An approach for the treatment of penile carcinoma. Cancer. 1977 Feb;39(2):456-66.

8. Catalano F, Trecca A, Rodella L, Lombardo F, Tomezzoli A, Battista $S$, et al. The modern treatment of early gastric cancer: our experience in an Italian cohort. Surg Endosc. 2009 Mar 5.

9. Catalano V, Labianca R, Beretta GD, Gatta G, de Braud F, Van Cutsem E. Gastric cancer. Crit Rev Oncol Hematol. 2009 Feb 19.

10. Cheng LY, Chen XD, Zhang YX, Feng XD. [Clinical significance of sentinel lymph node detection by combining the dye-directed and radioguided methods in gastric cancer]. Zhonghua Wai Ke Za Zhi. 2005 May 1;43(9):569-72.

11. Cheng LY, Xie ZY, Dai GR, Zhao WG. [Preliminary experiences of application of sentinel lymph node navigation technique in early gastric cancer]. Zhonghua Wai Ke Za Zhi. 2008 Jul 15;46(14):1076-9. 
12. Cheng LY, Zhong SZ, Huang ZH. Sentinel lymph node concept in gastric cancer with solitary lymph node metastasis. World J Gastroenterol. 2004 Oct 15;10(20):3053-5.

13. Chung IK, Lee JH, Lee SH, Kim SJ, Cho JY, Cho WY, et al. Therapeutic outcomes in 1000 cases of endoscopic submucosal dissection for early gastric neoplasms: Korean ESD Study Group multicenter study. Gastrointest Endosc. 2009 Feb 26.

14. Coburn NG. Lymph nodes and gastric cancer. J Surg Oncol. 2009 Mar 15;99(4):199-206.

15. Cochran AJ, Wen DR, Morton DL. Management of the regional lymph nodes in patients with cutaneous malignant melanoma. World J Surg. 1992 Mar-Apr;16(2):214-21.

16. El-Ghobashy $A E$, Saidi SA. Sentinel lymph node sampling in gynaecological cancers: techniques and clinical applications. Eur J Surg Oncol. 2009 Jul;35(7):675-85.

17. Gur AS, Unal B, Ozbek U, Ozmen V, Aydogan F, Gokgoz S, et al. Validation of breast cancer nomograms for predicting the nonsentinel lymph node metastases after a positive sentinel lymph node biopsy in a multi-center study. Eur J Surg Oncol. 2009 Jun 15.

18. Hayashi $H$, Ochiai $T$, Mori M, Karube T, Suzuki T, Gunji $Y$, et al. Sentinel lymph node mapping for gastric cancer using a dual procedure with dye- and gamma probe-guided techniques. J Am Coll Surg. 2003 Jan;196(1):68-74.

19. Ichikura T, Sugasawa H, Sakamoto N, Yaguchi Y, Tsujimoto H, Ono $\mathrm{S}$. Limited gastrectomy with dissection of sentinel node stations for early gastric cancer with negative sentinel node biopsy. Ann Surg. 2009 Jun;249(6):942-7.

20. Ichikura T. [Progress in sentinel node navigation surgery for gastric cancer]. Nippon Geka Gakkai Zasshi. 2009 Mar;110(2):68-72.

21. INCA INdC-. Estimativa de Incidência de Câncer no Brasil para 2008. Journal [serial on the Internet]. 2008 Date: Available from: http://www. inca.gov.br/estimativa/2008/index.asp?link=conteudo_view.asp\&ID=5

22. Ishikawa K, Yasuda K, Shiromizu A, Etoh T, Shiraishi N, Kitano S. Laparoscopic sentinel node navigation achieved by infrared ray electronic endoscopy system in patients with gastric cancer. Surg Endosc. 2007 Jul;21(7):1131-4.

23. Kitagawa $Y$, Fujii $H$, Mukai M, Kubota $T$, Ando $N$, Ozawa $S$, et al. Intraoperative lymphatic mapping and sentinel lymph node sampling in esophageal and gastric cancer. Surg Oncol Clin N Am. 2002 Apr;11(2):293-304.

24. Kitagawa Y, Fujii H, Mukai M, Kubota T, Otani Y, Kitajima M. Radioguided sentinel node detection for gastric cancer. Br J Surg. 2002 May;89(5):604-8.

25. Kitagawa Y, Kitajima M. Gastrointestinal cancer and sentinel node navigation surgery. J Surg Oncol. 2002 Mar;79(3):188-93.

26. Krag DN, Weaver DL, Alex JC, Fairbank JT. Surgical resection and radiolocalization of the sentinel lymph node in breast cancer using a gamma probe. Surg Oncol. 1993 Dec;2(6):335-9; discussion 40.

27. Lee JH, Ryu KW, Kim CG, Kim SK, Lee JS, Kook MC, et al. Sentinel node biopsy using dye and isotope double tracers in early gastric cancer. Ann Surg Oncol. 2006 Sep;13(9):1168-74.
28. Lee JH, Ryu KW, Nam BH, Kook MC, Cho SJ, Lee JY, et al. Factors associated with detection failure and false-negative sentinel node biopsy findings in gastric cancer: results of prospective single center trials. J Surg Oncol. 2009 Mar 1;99(3):137-42.

29. Morton DL, Wen DR, Wong JH, Economou JS, Cagle LA, Storm FK, et al. Technical details of intraoperative lymphatic mapping for early stage melanoma. Arch Surg. 1992 Apr;127(4):392-9.

30. Morton DL, Wong JH, Cagle LA. Lymphatic drainage of skin to a sentinel lymph node in a feline model. Ann Surg. 1991 Nov;214(5):637-41.

31. Nakajo A, Natsugoe S, Ishigami S, Matsumoto M, Nakashima S, Hokita S, et al. Detection and prediction of micrometastasis in the lymph nodes of patients with pNO gastric cancer. Ann Surg Oncol. 2001 Mar;8(2):158-62.

32. Nimura $H$, Narimiya $N$, Mitsumori $N$, Yamazaki $Y$, Yanaga $K$, Urashima M. Infrared ray electronic endoscopy combined with indocyanine green injection for detection of sentinel nodes of patients with gastric cancer. Br J Surg. 2004 May;91(5):575-9.

33. Ohdaira H, Nimura H, Mitsumori N, Takahashi N, Kashiwagi $\mathrm{H}$, Yanaga K. Validity of modified gastrectomy combined with sentinel node navigation surgery for early gastric cancer. Gastric Cancer. 2007; 10(2):117-22.

34. Oliveira AF, Oliveira Filho RS, Santos ID, Tucunduva TC, Sanches LG, Simoes e Silva Enokihara MM, et al. Sentinel lymph node biopsy in cutaneous melanoma. Acta Cir Bras. 2007 Sep-Oct;22(5):332-6.

35. Ott K, Herrmann K, Krause BJ, Lordick F. The Value of PET Imaging in Patients with Localized Gastroesophageal Cancer. Gastrointest Cancer Res. 2008 Nov;2(6):287-94.

36. Pinheiro LGP, Moraes MO, Soares AH, Lopes AJT, Naguére MASP, Brandão $C B$, et al. Estudo experimental de linfonodo sentinela na mama da cadela com azul patente e Tecnécio Tc99m. Acta Cir Bras [online]. 2003;18(6):545-52.

37. Torres de Melo JRM. Modelo Experimental em Caninos para Pesquisa de Linfonodo Sentinela no Estômago [Mestrado]. Fortaleza: Universidade Federal do Ceará; 2010.

38. Tsioulias GJ, Wood TF, Morton DL, Bilchik AJ. Lymphatic mapping and focused analysis of sentinel lymph nodes upstage gastrointestinal neoplasms. Arch Surg. 2000 Aug;135(8):926-32.

39. Veronesi U, Zingo L. [Surgical technic of breast amputation associated with axillary, internal mammary, supraclavicular and mediastinal lymph node excision]. Tumori. 1969 NovDec;55(6):413-6.

40. Winter A, Uphoff J, Henke RP, Wawroschek F. First Results of PET / CT-Guided Secondary Lymph Node Surgery on Patients with a PSA Relapse after Radical Prostatectomy. Aktuelle Urol. 2009 Jun 16.

41.Zulfikaroglu B, Koc M, Ozmen MM, Kucuk NO, Ozalp N, Aras G. Intraoperative lymphatic mapping and sentinel lymph node biopsy using radioactive tracer in gastric cancer. Surgery. 2005 Nov;138(5):899-904. 